INPLASY

PROTOCOL

To cite: Huang et al.

Acupuncture for adverse reactions after cesarean section:a protocol for systematic review and metaanalysis. Inplasy protocol

202070059. doi:

10.37766/inplasy2020.7.0059

Received: 15 July 2020

Published: 15 July 2020

Corresponding author:

WanPing Huang

851813721@qq.com

Author Affiliation:

Hubei University of Traditional

Chinese Medicine

Support: NA

Review Stage at time of this submission: The review has not yet started.

Conflicts of interest: NA.

\section{Acupuncture for adverse reactions after cesarean section:a protocol for systematic review and meta-analysis}

Huang, WP1; Fu, CW2; Deng, YR³ Wu, T4; Wang, HM5; Jiao, Y6.

Review question / Objective: The aim of this meta-analysis of randomized controlled trials is to summarize and evaluate evidence regarding the efficacy and safety of acupuncture for adverse reactions after cesarean section.

Condition being studied: Cesarean section is a common operation in obstetrics to save puerperae and perinatal infants.But it may lead to adverse reactions such as gastrointestinal dysfunction, pain and uroschesis. Acupuncture as a non-pharmacological option has been used in clinical practice. However, there is a lack of evidence to prove its effectiveness and safety.

Information sources: PubMed, Cochrane library, Web of Science, CINAHL(EBSCO), Embase, China National Knowledge Infrastructure(CNKI), Wanfang Database, VIP Database and China Biology Medicine(CBM) will be searched.

INPLASY registration number: This protocol was registered with the International Platform of Registered Systematic Review and Meta-Analysis Protocols (INPLASY) on 15 July 2020 and was last updated on 15 July 2020 (registration number INPLASY202070059).

\section{INTRODUCTION}

Review question / Objective: The aim of this meta-analysis of randomized controlled trials is to summarize and evaluate evidence regarding the efficacy and safety of acupuncture for adverse reactions after cesarean section.

Condition being studied: Cesarean section is a common operation in obstetrics to save puerperae and perinatal infants.But it may lead to adverse reactions such as gastrointestinal dysfunction, pain and uroschesis. Acupuncture as a nonpharmacological option has been used in clinical practice. However, there is a lack of evidence to prove its effectiveness and safety.

\section{METHODS}

Search strategy: PubMed, Cochrane Library, Web of Science, CINAHL(EBSCO), 
Embase, China National Knowledge Infrastructure(CNKI), Wanfang Database,VIP Dat abase and China Biology Medicine(CBM) will be searched from inception to June 2020. The key search terms are "Acupuncture OR Acupuncture Therapy OR Acupuncture, Ear OR electroacupuncture OR moxibustion OR acupressure" AND "Cesarean Section OR Cesarean Section, Repeat".

Participant or population: Women undergoing a cesarean section.

Intervention: Any intervention based on acupuncture-related therapies will be included, for instance, acupressure, moxibusion, transcutaneous electrical acustimulation and acupoint sticking. Acupunture theory combined other therapeutic methods such as traditional chinese medicine and metoclopramide, will also be adopted.

Comparator: Control group selecting usual care (means no treatment), sham acupuncture therapy, medication (metoclopramide, patient-controlled analgesia, neostigmine and so on).

Study designs to be included: Randomized controlled trials (RCTs) will be included.

Eligibility criteria: (1)Study type: Randomized controlled clinical trials (RCTS) for acupuncture therapy on adverse reactions after cesarean section, with or without a blind method;(2) Subjects: Puerpera meeting the clinical diagnostic criteria, of which there are no restrictions on age or source of cases, and the diagnostic criteria refer to the recognized clinical diagnostic criteria;(3) Intervention measures:The experimental group was treated by acupuncture-related therapies. (4) Outcome measures: Main outcomes are different according to adverse reactions. For example, the incidences of nausea and vomiting, the number of patients who need for antiemetic rescue, the total milk production and pain intensity.

Information sources: PubMed, Cochrane library,Web of Science, CINAHL(EBSCO),
Embase, China National Knowledge Infrastructure(CNKI), Wanfang Database, VIP Database and China Biology Medicine(CBM) will be searched.

Main outcome(s): Main outcomes are different according to adverse reactions.For example, the incidences of nausea and vomiting, the number of patients who need for antiemetic rescue, the total milk production and pain intensity.

Quality assessment / Risk of bias analysis: Cochrane risk-of-bias tool (ROB 2.0) will be used to evaluate the quality. Two reviewers will independently evaluated the quality of all matched studies. When disagreement appears, discussion will be initiated.A third author will request adjudications if necessary.

Strategy of data synthesis: Tables will be designed to summarize datas and show the key characteristics and important questions related to the aim of this review. STATA 14.0 will be used to perform pairwise meta-analysis. Addis 1.16.8, OpenBUGS 3.2.3 and STATA 14.0 will be used to conduct network meta-analysis.

Subgroup analysis: We conducted subgroup analysis for studies on the type of adverse reactions.

Sensibility analysis: A sensitivity analyses will be accomplished by excluding studies from the analysis one by one, if sufficient studies studies are available.

Language: Chinese and English.

Country(ies) involved: China.

Keywords: Acupuncture Therapy; Cesarean Section; adverse reaction; Systematic Review; Meta-Analysis.

Contributions of each author:

Author 1 - WanPing Huang.

Author 2 - ChengWei Fu.

Author 3 - Tong Wu.

Author 4 - YiRan Deng.

Author 5 - HuanMei Wang.

Author 6 - Yang Jiao. 